\title{
PROSPECÇÃO TECNOLÓGICA COMPARATIVA DE PATENTES DE PRODUTOS COSMÉTICOS UTILIZANDO METABÓLITOS SECUNDÁRIOS E PREPARAÇÕES COSMÉTICAS A PARTIR DO USO DE NANOTECNOLOGIA.
}

\author{
COMPARATIVE TECHNOLOLOGICAL PROSPECTION OF PATENTS OF COSMETIC \\ PRODUCTS USING SECONDARY METABOLITES AND COSMETIC PREPARATIONS \\ FROM THE USE OF NANOTECHNOLOGY
}

\author{
Josemeire dos Santos Machado ${ }^{1}$ \\ Juliana Azevedo da Paixão ${ }^{2}$
}

\begin{abstract}
RESUMO: Este trabalho tem como objetivo principal a busca de anterioridade sobre uso de metabólitos especiais empregados em preparações cosméticas, além de apresentar uma visão geral do estado atual de $\mathrm{P} \& \mathrm{D}$ de tecnologias nesta área de estudo. A metodologia de pesquisa inicial consistiu na associação dos dez diferentes códigos, Co7J71/oo, Co7G3,

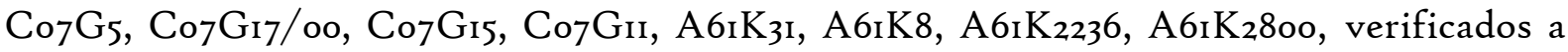
partir dos anos 1970 até 202I, associados a diferentes produtos naturais e a preparações farmacêuticas e cosméticas, posteriormente foram realizadas pesquisas comparativas do uso de nanotecnologia para a produção de cosméticos, utilizando os seguintes códigos:

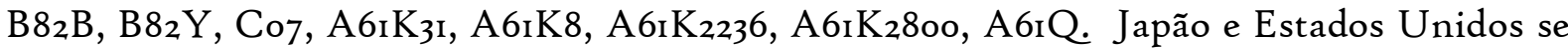
destacaram na área de cosméticos a partir de fontes naturais, com os maiores números de documentos de patentes depositados no grupo estudado, indicando que tais países estão seguros com a apropriação de seus conhecimentos. A partir da análise dos resultados encontrados na prospecção verificou-se que o uso de metabólitos secundários ainda é pouco difundido e apresentou um amplo crescimento entre os anos 1990 e 2000, apontando um decréscimo a partir de 2009 até a atualidade, demonstrando uma saturação do seu uso nesta área e a sua modificação por técnicas mais avançadas, como a nanotecnologia que exibiu crescimento a partir dos anos 200o, sendo O Japão, os Estados Unidos, Coréia e Rússia os principais detentores desta tecnologia.
\end{abstract}

Palavras-chave: Prospecção Tecnológica. Produtos Naturais. Cosméticos. Nanobiotecnologia.

ABSTRACT: This work has as main objective the search of previous use of special metabolites used in cosmetic preparations, besides presenting an overview of the current state of $\mid \mathrm{P} \& \mathrm{D}$ of technologies in this area of study. The initial research methodology consisted of the association of the ten different $\mathrm{Co}_{7} \mathrm{~J}_{7} / \mathrm{oo}_{\mathrm{O}} \mathrm{Co}_{7} \mathrm{G}_{3}, \mathrm{Co}_{7} \mathrm{G}_{5}, \mathrm{Co}_{7} \mathrm{G}_{17} /$ oo,

\footnotetext{
Graduação em Farmácia. Instituição de Ensino: Universidade Salvador (UNIFACS). Email:jm.machadook@gmail.com.

${ }^{2}$ Docente do curso de Farmácia. Professor DNSI Unifacs Instituição de ensino: Universidade Salvador (UNIFACS). E-mail: juliana.paixao@unifacs.br.
} 


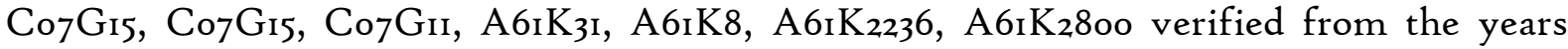
1970 to 2017 associated with different products natural and pharmaceutical and cosmetic preparations, comparative research on the use of nanotechnology for the production of cosmetics was carried out, using the following codes: $\mathrm{B} 8{ }_{2} \mathrm{~B}, \mathrm{~B} 82 \mathrm{Y}, \mathrm{Co} 7, \mathrm{~A} 6 \mathrm{I}_{3} \mathrm{~K}_{3}, \mathrm{~A} 6 \mathrm{I} \mathrm{K} 8$, A6IK2236, A6rK280o, A6rQ. Japan, the United States stood out in the area of cosmetics from natural sources, with the largest numbers of patent documents deposited in the group studied, indicating that such countries are safe with the appropriation of their knowledge. From the analysis of the results found in the prospection it was verified that the use of secondary metabolites is still not very widespread and presented a large growth between the years 1990 and 2000, showing a decrease from 2009 to the present, showing a saturation of its use in this area and its modification by more advanced techniques such as nanotechnology that showed growth from the 200os, with Japan, the United States, Korea and Russia being the main holders of this technology.

Keywords: Technological Prospecting. Natural products. Cosmetics. Nanotechnology.

\section{INTRODUÇÃO}

Produtos naturais são utilizados há milhares de anos pelas civilizações devido suas diversas propriedades, inclusive, terapêuticas. As plantas medicinais são detentoras de substâncias bioativas com elevado interesse econômico, essas substâncias podem ser pertencentes a várias classes químicas distintas, como alcaloides, terpenos, lignanas, flavonoides, cumarinas, benzenoides, quinonas, xantonas, lactonas e esteroides, entre outras (SIMÕES et al., 20I7).

Os metabólitos extraídos de plantas medicinais são amplamente utilizados nas indústrias farmacêuticas, alimentícias e de cosméticos. Os compostos fenólicos, por exemplo, apresentam elevada atividade antioxidante, anti-inflamatória, imunossupressão e proteção ultravioleta, podendo assim ser utilizados em um gama de produtos, sejam para uso farmacêutico ou cosmético (SOTO et al., 2015).

Desde o início da humanidade existe uma tendência da sociedade em modificar sua aparência, com o objetivo de melhorar a autoestima. Para isso, as diversas civilizações passaram a utilizar diferentes produtos seja de origem mineral, animal ou vegetal, com intuito de melhorar sua aparência, assim surgiram os produtos cosméticos (MÍNERO; DÍAZ, 2017). Com o advento da química orgânica e da síntese química as indústrias farmacêuticas e cosméticas passaram a ser a indústria com maior impacto econômico, e atualmente as terapias mais promissoras são aquelas obtidas a partir de moléculas desenvolvidas biologicamente com a finalidade de introduzir produtos uteis ao consumidor (DIAS; DE CARVALHO, 20I7).

Em busca do aperfeiçoamento físico-químico de substâncias bioativas para o desenvolvimento de formulações cosméticas, as indústrias utilizam estratégias de inovações tecnológicas, como a nanotecnologia. Os sistemas nano-estruturados são alvos constantes de pesquisas, essas nanoestruturas possibilitam a maior estabilização de substâncias com atividade cosmética, entre suas principais características pode-se ressaltar a alta superfície de contato, proteção de ativos sensíveis, redução de odores indesejáveis, alta tolerabilidade, capacidade de formação de filme sobre a pele, proteção contra 
degradação de substâncias, como também, podem evitar incompatibilidades entre os ingredientes da formulação. As nanoestruturas mais utilizadas em cosméticos são classificadas em nanocápsulas, nanoesferas, nanopartículas lipídicas sólidas, nanoemulsões, microemulsões, lipossomas e niossomas (DAUDT et al., 2013).

Nesse contexto, a prospecção tecnológica surge como uma ferramenta sistemática e objetiva, que tem como propósito o mapeamento de desenvolvimentos científicos e tecnológicos futuros, que influenciam significativamente uma indústria, a economia ou a sociedade como um todo (KUPFER; TIGRE, 2004; MALVEIRA et. al., 20I7). Assim, uma avaliação tecnológica significa identificar quais são as necessidades e as oportunidades futuras em um processo de pesquisa e desenvolvimento que sejam importantes na promoção e criação da capacidade de inovação de uma sociedade (MACHADO, 20I4).

A relevância das informações contidas em documentos de patentes tem tornado sua utilização mais comum. Empresas, instituições de pesquisas e desenvolvimento (P\&D) e universidades utilizam como indicador de desempenho econômico, pois o acesso às invenções propicia informações que não estão disponíveis em livros nem em artigos técnicos (SERAFINI et al., 2012). A grande maioria dos escritórios de patentes de cada país disponibiliza a informação através de uma página na internet. No Brasil as patentes estão disponíveis na base de dados do Instituto Nacional de Propriedade Intelectual (INPI), outros bancos de dados que indexam publicações de diversos países também estão disponíveis, como o Escritório Europeu de Patentes (Espacenet - EPO) (MAYERHOFF, 2008).

Diante do exposto o presente trabalho teve como objetivo realizar uma prospecção tecnológica na base de dados EPO, a fim de comparar a produção de patentes de produtos cosméticos utilizando metabólitos secundários com patentes de cosméticos utilizando nanotecnologia.

\section{METODOLOGIA}

A busca por documentos de patentes foi realizada de maneira a prover o maior número de documentos de patentes correspondentes ao tema de interesse, utilizando a classificação internacional de patentes que tornassem possível uma pesquisa representativa acerca da técnica de uso de metabólitos secundários como cosméticos, comparado ao uso da nanotecnologia. As tabelas i e 2 mostram o escopo utilizado para a pesquisa dos documentos de patentes.

Tabela I - Pesquisa por palavra-chave e por classificação internacional do uso de metabólitos secundários em produtos farmacêuticos e cosméticos

\begin{tabular}{|c|c|c|c|c|c|c|c|c|c|c|c|}
\hline Co7J71/oo & $\mathrm{Co}_{7} \mathrm{G}_{3}$ & $\mathrm{Co}_{7} \mathrm{G}_{5}$ & $\mathrm{Co7}_{7} \mathrm{Gi7}_{\text {/oo }}$ & $\mathrm{Co7}_{075}$ & $\mathrm{Co7}_{\mathrm{GII}}$ & $\mathrm{A}_{61} \mathrm{~K}_{3 \mathrm{I}}$ & A6IK8 & $\mathrm{A}_{61 \mathrm{I}_{2236}}$ & $\mathrm{~A}_{61 \mathrm{~K} 2800}$ & A6rQ & $\begin{array}{l}\text { World } \\
\text { Wide }\end{array}$ \\
\hline $\mathrm{x}$ & & & & & & $\mathrm{x}$ & & & & & 8.976 \\
\hline \multirow[t]{8}{*}{$\mathrm{x}$} & & & & & & & $\mathrm{x}$ & & & & 194 \\
\hline & $\mathrm{x}$ & & & & & $\mathrm{x}$ & & & & & 756 \\
\hline & $\mathrm{x}$ & & & & & & $\mathrm{x}$ & & & & 173 \\
\hline & & $\mathrm{x}$ & & & & $\mathrm{x}$ & & & & & 164 \\
\hline & & $\mathrm{x}$ & & & & & $\mathrm{x}$ & & & & 6 \\
\hline & & & $\mathrm{x}$ & & & $\mathrm{x}$ & & & & & 419 \\
\hline & & & $\mathrm{x}$ & & & & $\mathrm{x}$ & & & & 198 \\
\hline & & & & $\mathrm{x}$ & & $\mathrm{x}$ & & & & & $3 \mathrm{I}$ \\
\hline
\end{tabular}




\begin{tabular}{|c|c|c|c|c|c|c|c|c|c|c|c|}
\hline & & & & & $x$ & $\mathrm{x}$ & & & & & 2130 \\
\hline & & & $\mathrm{x}$ & & & $x$ & & $\mathrm{x}$ & & & 0 \\
\hline & & & $\mathrm{x}$ & & & & $x$ & & $\mathrm{x}$ & & o \\
\hline$x$ & & . & & & & & & & & $\mathrm{X}$ & 150 \\
\hline & $\mathrm{x}$ & & & & & & & & & $\mathrm{X}$ & 164 \\
\hline & & $x$ & & & & & & & & $\mathrm{X}$ & 6 \\
\hline & & & $\mathrm{x}$ & & & & & & & $\mathrm{X}$ & 173 \\
\hline & & & & $\mathrm{x}$ & & & & & & $\mathrm{X}$ & 3 \\
\hline & & & & & $x$ & & & & & $\mathrm{X}$ & 17 \\
\hline
\end{tabular}

Fonte: Autoria própria, 2021.

- Co7J7i/oo Esteroides em que o esqueleto de ciclopentanoperidrofenantreno é condensado com um anel heterocíclico (anéis heterocíclicos espiro - condensados Co7J21/oo, Co7J33/oo, Co7J43/oo)

- $\mathrm{Co7G}_{17} /$ oo- Outros compostos de constituição desconhecida

- $\mathrm{Co}_{7} \mathrm{G}_{5}$-Alcaloides

- $\mathrm{Coy}_{3}$ - Glicosídeos (Polissacarídeos Co8B)

- Co7Gis-Hormônios

- Co7Gir-Antibióticos

- $\mathrm{A} 6 \mathrm{I} \mathrm{K}_{31-}$ - Preparações medicinais contendo ingredientes ativos orgânicos

- A6rK8- Produtos cosméticos ou semelhantes de toalete (invólucros ou acessórios para armazenamento ou manuseio de produtos de toalete sólidos ou pastosos ou substâncias cosméticas $\mathrm{A}_{45} \mathrm{D}_{40} \mathrm{o}$ oo

- A6iK2236- Métodos de isolamento ou extração de preparações medicinais de constituição indeterminada contendo material de algas, líquenes, fungos ou plantas ou seus derivados por exemplo: fitoterapia tradicional

- A6rK28oo- Propriedades de composição cosméticas ou ingredientes ativos das mesmas ou auxiliares de formulação usados nas mesmas e aspectos relacionados ao processo

Tabela 2: Pesquisa por palavra-chave e por classificação internacional do uso de nanotecnologia para produção de cosméticos.

\begin{tabular}{|c|c|c|c|c|c|c|c|c|}
\hline B82B & B82Y & $\mathrm{Co} 7$ & $\mathrm{~A} 6 \mathrm{IK}_{3 \mathrm{I}}$ & A6rK8 & $\mathrm{A}_{61 K_{2236}}$ & $\mathrm{~A}_{61 K_{28} 800}$ & $\mathrm{~A} 6 \mathrm{rQ}$ & World wide \\
\hline $\mathrm{x}$ & & & $\mathrm{x}$ & & & & & 825 \\
\hline $\mathrm{x}$ & & $\mathrm{x}$ & $\mathrm{x}$ & & & & & 93 \\
\hline $\mathrm{x}$ & & $\mathrm{x}$ & & & $\mathrm{x}$ & & & o \\
\hline $\mathrm{x}$ & & & & & & & $\mathrm{x}$ & 173 \\
\hline $\mathrm{x}$ & & $\mathrm{x}$ & & & & & $\mathrm{x}$ & II \\
\hline $\mathrm{x}$ & & $\mathrm{x}$ & & & & $\mathrm{x}$ & & o \\
\hline $\mathrm{x}$ & & $\mathrm{x}$ & & $\mathrm{x}$ & & & & 14 \\
\hline \multirow[t]{8}{*}{$\mathrm{x}$} & & & & $\mathrm{x}$ & & & & 221 \\
\hline & $\mathrm{x}$ & & $\mathrm{x}$ & & & & & $<$ <I0.000 \\
\hline & $\mathrm{x}$ & $\mathrm{x}$ & $\mathrm{x}$ & & & & & 5.111 \\
\hline & $\mathrm{x}$ & & & $\mathrm{x}$ & & & & 7.311 \\
\hline & $\mathrm{x}$ & $\mathrm{x}$ & & $\mathrm{x}$ & & & & 487 \\
\hline & $\mathrm{x}$ & $\mathrm{x}$ & & & $\mathrm{x}$ & & & 0 \\
\hline & $\mathrm{x}$ & $\mathrm{x}$ & & & & $\mathrm{x}$ & & o \\
\hline & $\mathrm{x}$ & $\mathrm{x}$ & & & & & $\mathrm{x}$ & 437 \\
\hline
\end{tabular}


Fonte: Autoria própria, 2021.

- B82B - Nanopartículas

- $\mathrm{Co} 7$ - Química orgânica

- $\mathrm{A} 6 \mathrm{IK}_{31}$ - Preparações medicinais contendo ingredientes ativos orgânicos

- A6IK8 - Preparações cosméticas ou semelhantes de toalete (invólucros ou acessórios para armazenamento ou manuseio de produtos de toalete sólidos ou pastosos ou substâncias cosméticas $\mathrm{A}_{45} \mathrm{D}_{40}$ /oo

- A6iK2236 - Métodos de isolamento ou extração de preparações medicinais de constituição indeterminada contendo material de algas, líquenes, fungos ou plantas, ou seus derivados, por exemplo: fitoterapia tradicional

- A6IK280o - Propriedades de composições cosméticas ou ingredientes ativos das mesmas ou auxiliares de formulação usados nas mesmas e aspectos relacionados ao processo.

As buscas foram realizadas por meio do banco de patentes, do International Patent Classification (IPC), o ESPACENET, que é uma base mundial de acesso livre usualmente empregada em trabalhos de prospecção. A coleta de dados foi realizada em maio de 202I, não houve limitação de período, objetivando mapear documentos de patente sobre o uso de metabólitos secundários em cosméticos e uso de nanotecnologia para produção de cosméticos. A metodologia consistiu na associação dos seguintes códigos Co7J71/oo,

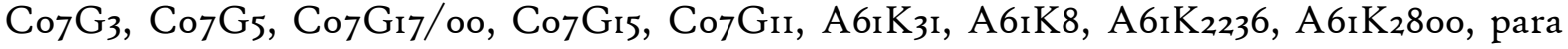
identificação do número de patentes e sua evolução sobre o uso dos metabólitos secundários em produtos de beleza, posteriormente foram realizadas novas associações com os seguintes códigos: $\mathrm{B} 82 \mathrm{~B}, \mathrm{~B} 82 \mathrm{Y}, \mathrm{Co} 7, \mathrm{CA} 6{ }_{1 \mathrm{~K}}{ }_{31}, \mathrm{~A} 61 \mathrm{~K} 8, \mathrm{~A} 6 \mathrm{IK}_{2236}, \mathrm{~A} 6 \mathrm{I}_{2} \mathrm{~K}_{200}$ a fim de identificar a evolução do uso de nanotecnologia na produção de cosméticos à base de compostos orgânico, os dois escopos foram comparados entre si. A base de dados escolhida foi a European Patent Office (EPO), uma base mundial de acesso livre usualmente escolhida para prospecção com patentes de mais de 90 países. As patentes utilizadas como base para pesquisa estão sinalizadas em amarelo nas tabelas i e 2, o critério de uso foi a maior especificidade para área de cosméticos.

\section{RESULTADOS E DISCUSSÃO}

Foram encontradas 57ipatentes desenvolvidas a partir de metabólitos secundários que foram exportadas da base de patentes EPO para Excel, onde foram tratados os dados, retirando repetições efetuando limpeza, restando 262 documentos para serem analisados. Estes resultados mostraram que existem relativamente poucas patentes depositadas relacionadas ao uso destes metabólitos essenciais como fonte de novos produtos cosméticos. Posteriormente foram analisados o número de patentes a partir do uso de nanotecnologia para a produção de produtos cosméticos e foram encontradas 1.343 patentes desenvolvidas a partir desta tecnologia, os dados foram exportados para o programa Excel e tratados, retirando as repetições e efetuando a limpeza, restando 348 arquivos relevantes para serem analisados.

A Figura I mostra uma comparação da evolução dos números de patentes por ano de publicação entre patentes de cosméticos a partir de metabólitos especiais e patentes de cosméticos a partir de nanotecnologias. Em relação ao depósito de patentes por ano pelo 
uso de metabólitos secundários, houve um baixo número de patentes na década de 80 até o final da década de 90 , em que a média de patentes depositadas foi de aproximadamente quatro patentes por ano, sendo que no período de 1970 a 1979 houve depósito de 2i patentes. Esse número começou a crescer no início da década de 9o, com um aumento progressivo do número de patentes por ano até início dos anos 2000. Entre os anos 2001 e 2015 observou-se um decréscimo significativo que pode ser justificado pelo aumento de novas tecnologias, como o uso de lipossomas, nano-emulsões e nano-partículas.

Ainda de acordo com a figura I é possível verificar um aumento progressivo do número de patentes depositadas a partir da produção de cosméticos pelo uso de nanotecnologia a partir do momento do decréscimo do uso dos metabólitos secundários, justificando assim a queda dos números de patentes do mesmo. A partir dos anos $2000 \mathrm{o}$ uso de nanotecnologia passou a dominar no desenvolvimento de novos produtos cosméticos, entre os anos de 2001 até 2015 a média do número de patentes foi de aproximadamente 20 patentes por ano, tendo seu auge entre 2006 a 2012, ano ao qual é possível afirmar que a tecnologia entrou em maturidade, sendo considerada uma tecnologia chave , segundo Graff, (2012) uma tecnologia chave é definida por estar em uma posição central da base de conhecimento, tendo a função de uma fonte de novidade.

Os compostos bioativos são fontes importantes para a produção de produtos farmacêuticos e cosméticos, pesquisas demostram q a maior parte dos medicamentos foram desenvolvidos direta ou indiretamente a partir de fontes naturais (CRAGG; NEWMAN, 2013). Muitos ativos naturais são compostos instáveis, dessa maneira podem sofrer reações que levam à diminuição ou perda de eficácia e degradação do produto final. Necessitando do conhecimento de novas tecnologias que melhorem o desempenho destes produtos e a sua aceitação pelo consumidor (DAUDT, 2013). Tais problemas relacionados ao uso destes bioativos incentivou o desenvolvimento de novas tecnologias, como alternativa para aumentar a estabilidade, como também permitir a liberação controlada das substâncias ativas. Uma alternativa importante e que mudou o cenário da produção de cosméticos apenas utilizando metabólitos secundários foi o uso do encapsulamento destas substâncias ativas através de técnicas que envolvem a nanotecnologia.

De acordo Cotinguiba, (2013) as formulações farmacêuticas/ cosméticas conhecidas utilizam substâncias sintéticas ou extratos vegetais, inseridos numa formulação, o que torna tal produto altamente instável. Processos que proporcionem o aprisionamento destas substâncias seriam importantes para aumentar a estabilidade dos produtos finais, como por exemplo o uso de nanoemulsões, lipossomas. Dessa forma, prolongando a estabilidade do produto.

De acordo com dados coletados na base de dados Web of Science, o número de publicações incluindo concomitantemente o radical "nano" e a palavra "skin" aumentou I2 vezes desde a década de 90 até os anos 2000 , sendo que só no ano de 2012 foram mais de mil artigos publicados envolvendo o tema (DAUDT, 2013). E o uso desta técnica em produtos naturais está atrelada principalmente a estabilidade dos produtos, bem como o melhor aproveitamento pelo consumidor. 
Figura I - Comparação da evolução anual do depósito de patentes conforme escopo deste trabalho: Número de patentes de metabolitos secundários utilizados em cosméticos por ano e número de patentes de cosméticos utilizando nanotecnologia por ano.

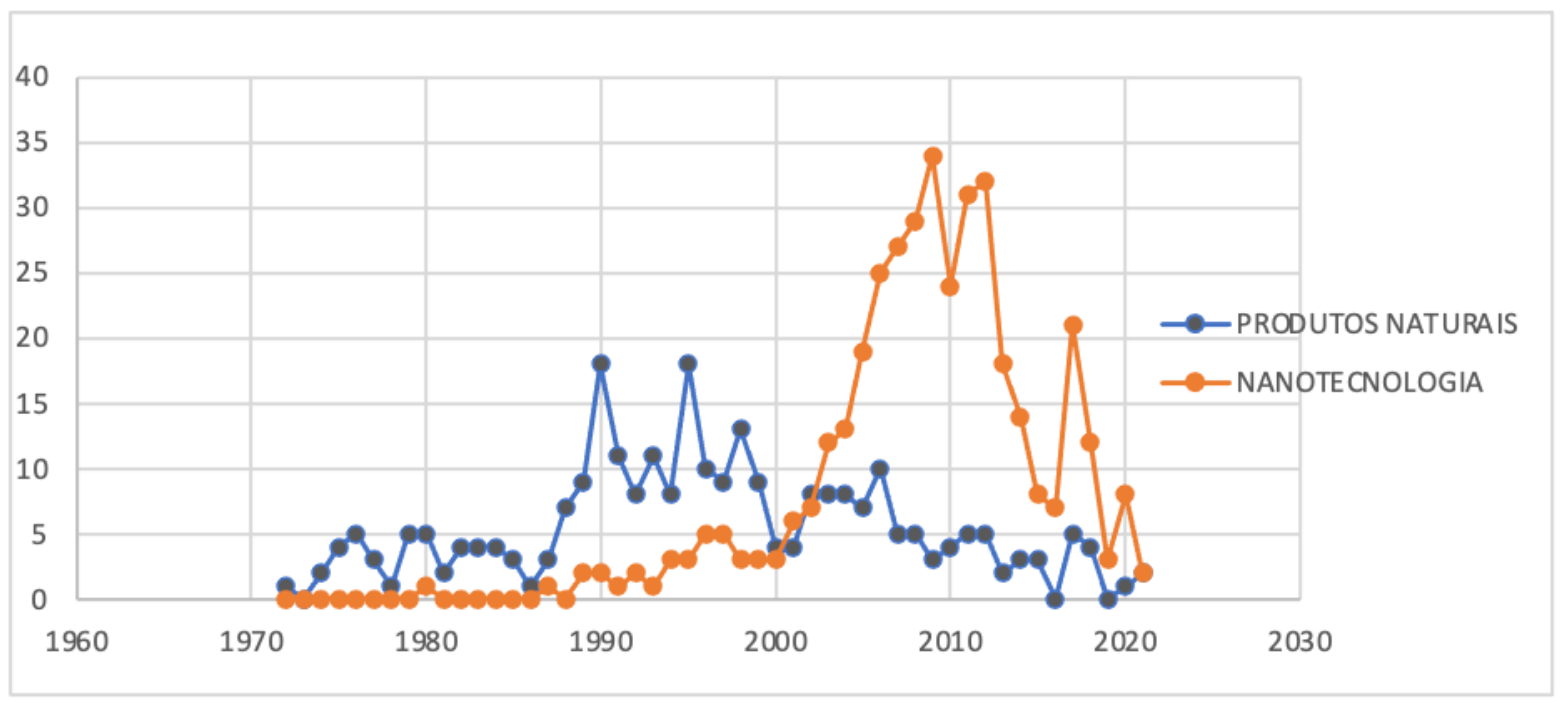

Fonte: Autoria própria, 202I.

Com relação ao número de patentes depositadas pelo uso de metabólitos secundários os países Japão e EUA são os maiores detentores da tecnologia relativa à produção de cosmético, como mostrado na Figura 2. O Brasil aparece apenas com I patente nesse período, estando entre os últimos no ranking. Em relação ao número de patentes por país do uso de nanotecnologia em cosméticos, os EUA, Coréia do sul, Japão e Rússia são os 4 países com maior número de depósito de patentes, 99, 89, 58 e 35 respectivamente. A Coreia do Sul mostrou grande importância no depósito de patentes na área de nanotecnologias em cosméticos, já que a quantidade de patentes depositadas neste campo foi muito superior ao número de patentes relacionadas ao uso de metabólitos especiais, 89 patentes versus 5 patentes, assim como a Rússia não apresentou nenhum depósito a partir do uso de metabólitos, no entanto depositou 35 patentes com o uso de novas tecnologias. $\mathrm{O}$ Brasil está situado na oitava posição, junto com África do Sul, com 7 patentes cada.

Estudos mostram que países como Japão, Estados Unidos e França são os principais no ranking mundial no ramo de cosméticos (ABDI, 20II). Segundo Ramos et. al, 20o8, quem detém o maior nível de conhecimento nesta área são Estados Unidos, União Europeia e Japão, que investem bilhões na produção de cosméticos e desenvolvimentos de novas tecnologias como a nanotecnologia, concentrando juntos cerca da metade dos investimentos no mundo. Porém países como Rússia, Coreia, China e outros também têm realizado investimentos significativos em redes de pesquisa e projetos na área de nanotecnologia.

Conforme Tomazzoni et al., (2006) de 1999 para 200o, as vendas de fitoterápicos aumentaram 15\%, contra $4 \%$ dos medicamentos sintéticos e já atingem US $\$ 260$ milhões/ano, Paraná, São Paulo, Bahia, Maranhão, Amazonas, Pará e mato Grosso são os maiores exportadores de plantas medicinais, principalmente para países como: Estados 
Unidos, Alemanha, Países Baixos, França, Japão, Portugal, Itália, Coréia do Sul, Reino Unido, Espanha, Suíça e Australia. Como também Emiliano e Balliano (2019) na última década as plantas medicinais têm se destacado como produto de valor agregado, o número de países que importam essas matéria-prima vem se ampliando compreendendo países como Alemanha, Estados Unidos, Singapura, Malásia e Japão, estes os maiores importadores de plantas medicinais e aromáticas. Tais fatos confirmam a precedência dos EUA, Japão como os maiores detentores da tecnologia.

Figura 2 - Países que mais detém patentes na área em estudo: Número de patentes de metabolitos secundários utilizados em cosméticos por país e número de patentes de cosméticos utilizando nanotecnologia por país.

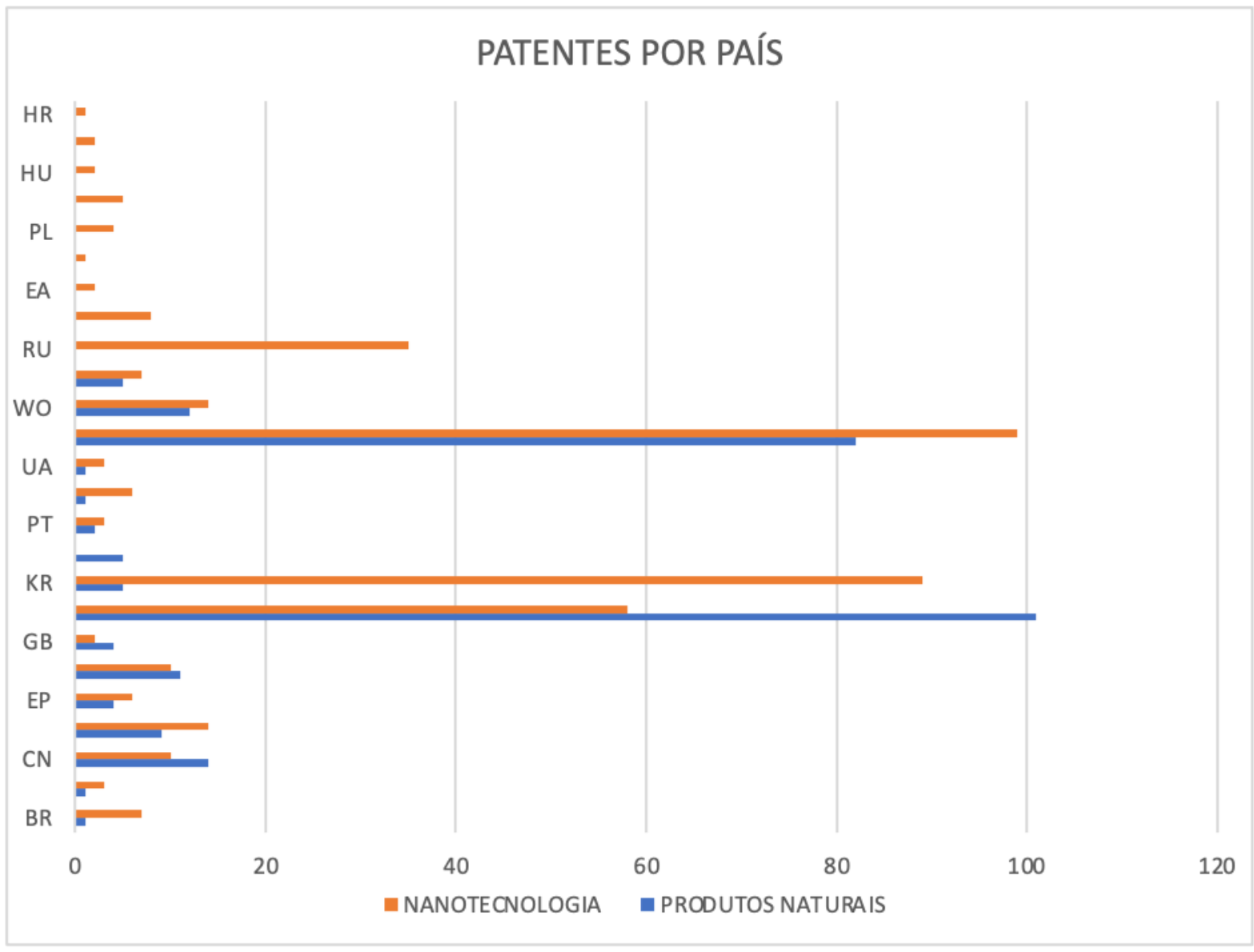

Fonte: Autoria própria, 2021.

Com relação aos inventores mais produtivos, os que se destacam estão listados na figura 3, o que apresenta maior número de patentes foi O'lenick Jr Anthony dos Estados Unidos, com 6 patentes depositadas. Nenhum dos primeiros titulares com maior número de patentes apresentou patentes nos dois campos de atuação. No entanto, esses inventores vêm desenvolvendo patentes em parcerias com diversos outros. Os inventores mais produtivos estão alocados nos países com maior número de depósitos. 
Figura 3 - Principais titulares por patente depositada.

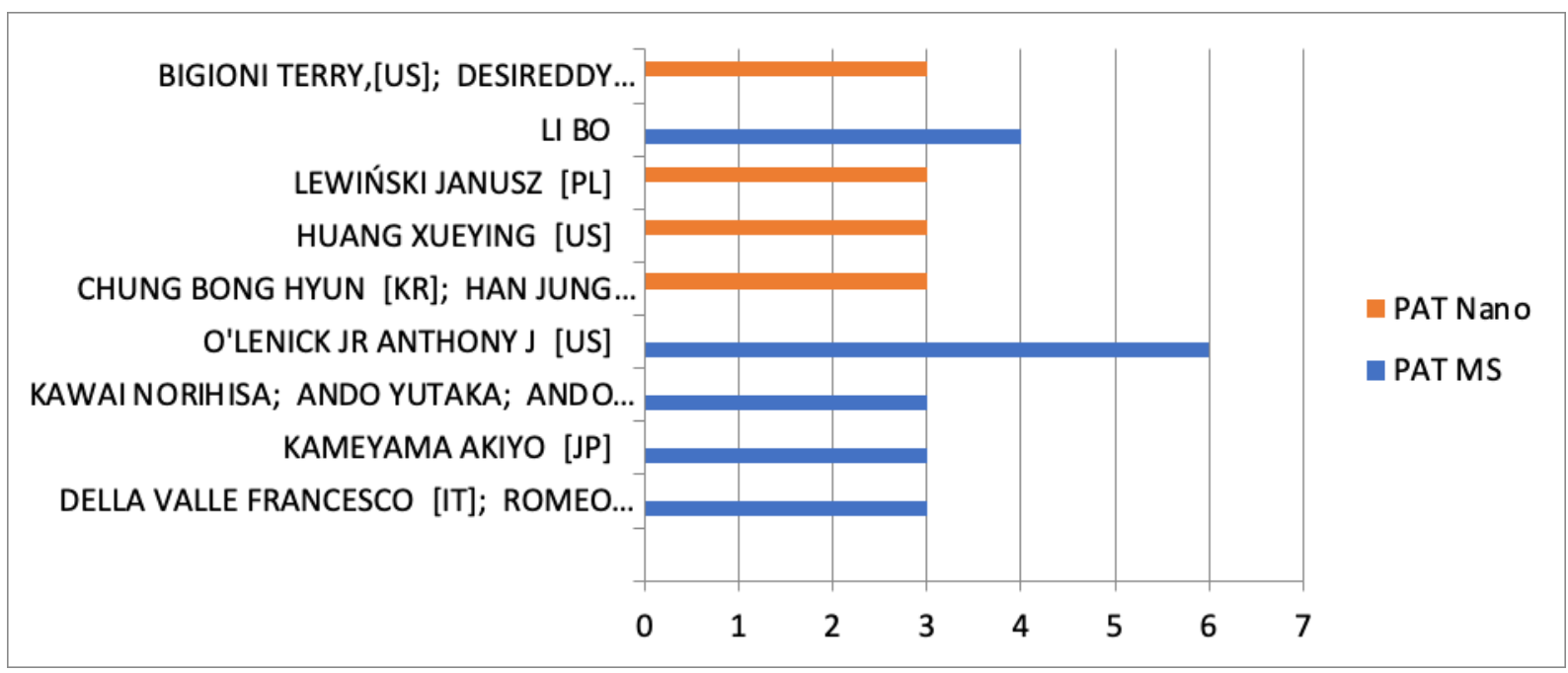

Fonte: Autoria própria, 2021

Os códigos de classificação internacional mais relevantes estão listados na figura 4, os códigos que aparecem mais vezes são os seguintes: A6ıK8/97, A6ıK8/6o, A6ıK8/73, Co7J7i/oo.

Figura 4 - Códigos de classificação Internacional mais relevantes dos documentos depositados

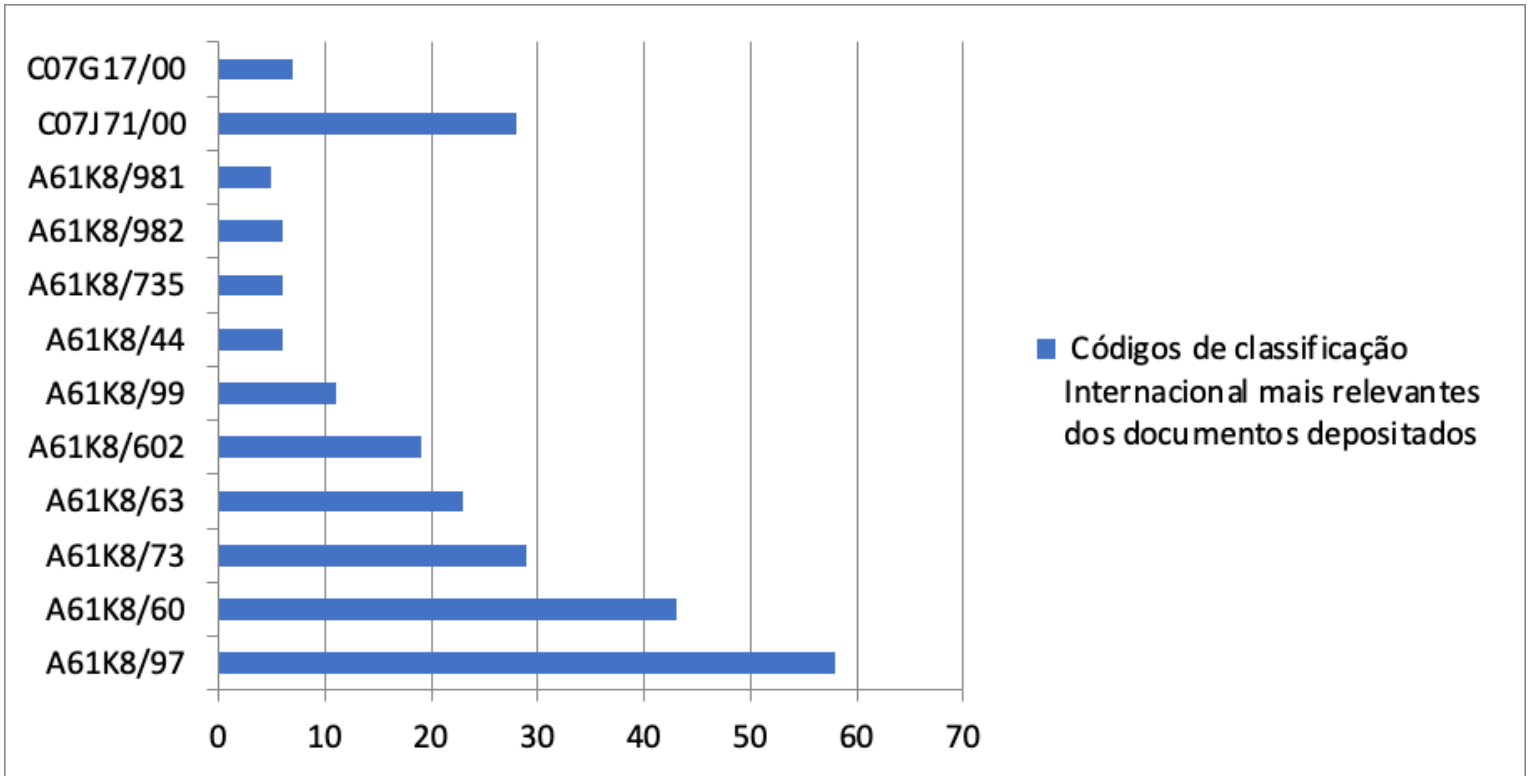

Fonte: Autoria própria, 2021.

A figura 5 apresenta as empresas maiores depositantes para as duas áreas de atuação. As seguintes empresas Henkel Corp [US], Ichimaru Pharcos INC, Kao Corp [JP], Nestle AS [CH] e Oreal [FR], apresentam o maior número de depósitos de patentes de cosméticos oriundos de metabólitos secundários, enquanto as empresas Amorepacific Corp [KR] e Oreal [FR] são os destaques do uso de nanotecnologias em cosméticos. No 
entanto a Oreal [FR] está em destaque nas duas vertentes com 7 patentes para metabólitos secundários e 13 patentes para o uso de nanocosméticos. É importante notar que tais empresas estão inseridas nos países que lideram o ranking de depósitos de patentes, exceto a Oreal [FR] que está localizada na França. O Brasil não aparece no gráfico, no entanto uma empresa muito importante, a Natura, apresenta 2 patentes depositadas.

L'Oréal é um grupo francês de cosméticos, a empresa foi fundada por Eugène Schueller em 1909, atualmente é um grupo internacional e líder mundial na indústria de cosméticos (L'Oreal, 2017). Os dados encontrados demonstram o tamanho da liderança da L'Oreal no ramo de cosméticos, dominando nos dois campos de metabólitos secundários e nanocosméticos. Entre os anos de 1994 e 2005, a L'Oreal foi a quinta empresa no mundo com relação ao número de patentes depositadas na área de nanotecnologia (MIHRANYAN, 2012).

Figura 5 - Principais empresas depositantes de patentes.

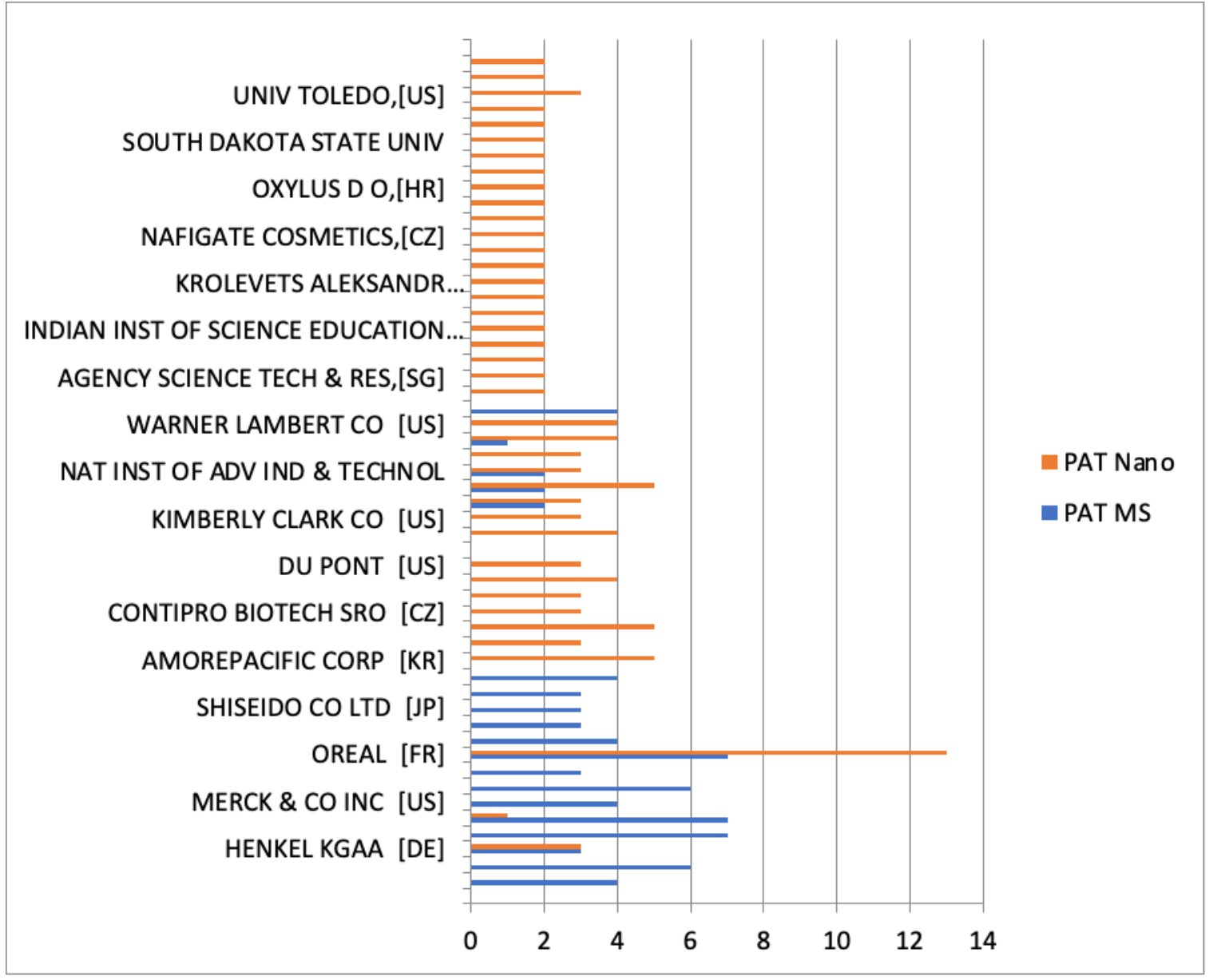

Fonte: Autoria própria, 2021.

Uma patente depositada no ano de 200I pela L'Oreal [FR] com número US6384025 B2, refere-se a uma nova formulação de derivados de retinol, tais produtos podem ser utilizados para o combate a acne e ao envelhecimento da pele e cabelos. Já a patente de número USoo57736ır A depositada em 1997 com título: Ceramidas., processo de preparação 
e sua aplicação no cosmético e campos dermopharmacéutico, refere-se a processos de preparo de ceramidas para serem utilizadas em produtos para pele ou cabelo com danos solares ou outros danos. É possível verificar que ambas as patentes tratam de produtos destinados ao tratamento do desgaste por danos ao cabelo ou a pele, tais produtos tem grande apelo de mercado, tendo em vista que a indústria da beleza, mostra que existem formulações farmacêuticas e cosméticas capazes de neutralizar a ação dos radicais livres seja para uso interno, diminuindo assim os efeitos da idade.

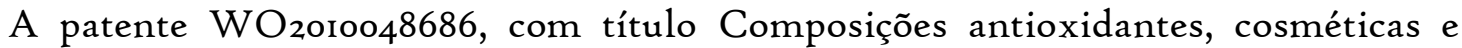
farmacêuticas que contêm complexos de substâncias, descreve o processo de combinações de extratos de plantas com atividade antioxidante inseridos em formulações cosméticas, tendo a vantagem de estas combinações apresentarem atividade sinérgica, o que potencializa a ação antioxidante e atividade anti-idade.

Todas as patentes citadas acima apresentam a desvantagem de deixarem os constituintes expostos a processos oxidativos, já que não existe nenhuma forma de proteção ou encapsulação dos extratos antes da formulação. As patentes que ganharam lugar atualmente, são as patentes que envolvem microencapsulação, com o objetivo de manter a integridade dos produtos. Segundo Aranha, (2015), o processo de microencapsulação, emulsões ou lipossomas, aumentam a estabilidades de substâncias antioxidantes a variadas condições adversas, como a presença de luz, $\mathrm{pH}$ e outros, estabilizando o produto final. Além de proporcionar uma melhor absorção dos antioxidantes na pele, devido a aumentar a estabilidade das substâncias prolongando o tempo de contato com a pele.

Uma patente interessante utilizando a técnica de nanoparticulas é a intitulada Emulsions anti-uv stabilisees avec de la lignine et des nanoparticules, de número FR3044239 (AI), tal patente refere-se ao uso de emulsões com atividade anti-idade e antiUV que são estabilizadas pela presença de nanopartículas de ligninas. Outra patente interessante é a intitulada Nanoemulsion comprising ginseng saponin metabolite as active ingredient, method for producing the same and cosmetic composition for preventing skin aging containing the same, de número JP4549625 (B2); JP2003212776 (A), a mesma também utiliza de produtos naturais microencapsulados com o objetivo de diminuir o envelhecimento da pele.

O envelhecimento da população tem influenciado a busca de novas tecnologias que possibilitem a continuidade da beleza. E é importante observar que as pesquisas que envolvem prospecção tecnológica são de extrema importância para o mercado, tendo em vista que identifica as mudanças mercadológicas que podem ser influenciadas por diversos parâmetros.

\section{CONCLUSÃO}

A Prospecção Tecnológica mostrou que o uso da técnica de nanotecnologia em produtos cosméticos superou a produção de cosméticos a base de produtos naturais, tendo o seu grande salto em meados do século XXI, destacando os Estados Unidos, Japão, Coreia como os maiores detentores da tecnologia. Já o Brasil ainda apresenta um número reduzido 
de patentes, sendo este assunto promissor para investimentos de pesquisa, desenvolvimento e apropriação tecnológica. Este estudo mostrou que é possível efetuar um levantamento de uma quantidade considerável de patentes depositadas sobre o tema proposto, bem como realizar um panorama de anterioridade sobre a situação de $P \& D$ de determinado setor industrial.

\section{REFERÊNCIAS}

AGENCIA BRASILEIRA DE DESENVOLVIMENTO INDUSTRIAL (ABDI). Estudo Prospectivo de Nanotecnologia. Brasília: ABDI, 2010.

ABDI, Abihpec, Sebrae, II Caderno de Tendências. Higiene Pessoal, Perfumaria e Cosméticos, Vol. 2. 20Ir.

ARANHA CPM. Microencapsulação por gelificação iônica e interação eletrostática do corante de buriti (Mauritia flexuosa L. f.). [Tese de Doutorado], 2015.

COTINGUIBA GG. Método de Avaliação da Defesa Antioxidante: Uma Revisão de Literatura. UNOPAR Científica Ciências Biológicas e da Saúde, v.15, n.3, 2013.

CRAGG, G. M.; NEWMAN, D. J. Natural products: a continuing source of novel drug leads. Biochimica biophysica acta. V. 1830, N. 6, P. 3670-3695, 2013.

DAUDT, R. M.; EMANUELLI, J.; KÜLKAMP-GUERREIRO, I. C.; POHLMANN, A. R.; GUTERRES, S. S. A nanotecnologia como estratégia para o desenvolvimento de cosméticos. Ciência e Cultura, v.65, n.3, 2013.

DIAS, R. F.; DE CARVALHO, C. A. A. Bioeconomia no Brasil e no Mundo: Panorama Atual e Perspectivas Ver. Virtual Quim. V. 9, n. I, 2017.

EMILIANO, Sannyele Alcantara; BALLIANO, Tatiane Luciano. Prospecção de Artigos e Patentes sobre Plantas Medicinais Presentes na Caatinga Brasileira. Cadernos de Prospecção, v. 12,n. 3, p. 615, 2019.

GRAFF, H. Inventor Networks in Emerging Key Technologies: Information Technology vs. Semiconductors. J Evol Econ 22:459-48o, 2012.

HAMBARDZUMYAN ARAYIK [FR]. FR3044239. Emulsions anti-uv stabilisees avec de la lignine et des nanoparticules. 02 de junho de 2017.

KUPFER, D.; TIGRE, P. Prospeç̧ão Tecnológica - Modelo Senai de Prospecção documento metodológico [Cap.2]. Montevidéu: OIT/CINTERFOR. 2004.

L’Oreal. Loreal group. http://www.loreal.com/, 2017.

MACHADO, BRUNA APARECIDA SOUZA, et al. "Tendências tecnológicas de embalagens biodegradáveis através da prospecção em documentos de patentes." Cadernos de Prospecção 5.3 (2014): 132. 
MALVEIRA S.; Santos L. F., Tormim F. S. P.; Souza D. N., Godinho L. C. S.; Nascimento P. G. B. D.; Ghesti G. F. Estudo prospectivo sobre equipamentos médicos de ablação por radiofrequência. Cad. Prospec., Salvador, v. Io, n. 4, 2017.

MAYERHOFF, Z.D.V.L. Uma análise sobre os estudos de prospecção tecnológica. Cadernos de prospecção, v. I, n. I, p. 7-9, 2008.

MIHRANYAN, A.; Ferraz, N.; Strømme, M. "Current status and future prospects of nanotechnology in cosmetics”. Progress in Materials Science, vol. 57, nㅇ875. 2012.

MINERO, F. J. G.; DÍAZ, L. B. História y actualidad de productos para la piel, cosméticos y fragancias. Especialmente los derivados de las plantas. Ars Pharmaceutica, v.58, n.I, p.5-12, 2017.

RAMOS, B. G. Z; PASA, T. B. C. O desenvolvimento da nanotecnologia: cenário 54 Visão Acadêmica, Curitiba, v.I3, n.I, Jan. - Mar./2012 - ISSN 1518-5192 mundial e nacional de investimentos. Rev. Bras. Farm., v. 89 (2): p. 95-IoI, 2008.

SERAFINI, M.R.; QUINTANS, J. S.; ANTONIOLLI, A. R.; SANTOS, M. R. V.; QUINTANS JUNIOR, L. J. Mapeamento de tecnologias patenteáveis com o uso da hecogenina. Revista Geintec, v. 2, n. 5, p. 427- 435, 2012.

SIMÕES, C.M.O.; SCHENKEL, E.P.; GOSMANN, G.; MELLO, J.C.P.; MENTZ, L.A.; PETROVICK. P.R.(Orgs). Farmacognosia: do produto natural ao medicamento. Porto Alegre: artemed, 2017.

SOTO, M. L.; FALQUÉ, E.; DOMÍNGUEZ, H. Relevance of Natural Phenolics from Grape and Derivative Products in the Formulation of Cosmetics. Cosmetics, v.2, p.259276, 2015 .

TOMAZZPNI, Marisa Ines; NEGRELLE, Raquel Rejane Bonato; CENTA, Maria de Lourdes. Fitoterapia popular: a busca instrumental enquanto prática terapeuta. Texto \& Contexto-Enfermagem, v. I5, n. I, p. II5-I21, 2006.

TULOUP REMY; DALKO MARIA [FR]; RUBINSTENN GILLES US6384025. Retinyl carbonate derivatives, preparation process and uses. 2001, 02 de abril de 2002.

YOO BYUNG HEE; KANG BYUNG YOUNG; YEON MYEONG HOON; SUN JU HEE KYUNG; KWON SUN SANG; KIM HANKON. Nanoemulsion con saponin metabolite as active ingredient, method for producing the same and cosmetic preventing skin aging containing the same. JP4549625; JP2003212776. 30 de julho de 2003

ZYSMAN ALEXANDRE; VANLERBERGHE GUY; SEMERIA DIDIER USoo57736ri. Ceramides, process for their preparation and their applications in the cosmetic and dermopharmaceutical fields. 26/02/1997. 30 de junho de 1998. 